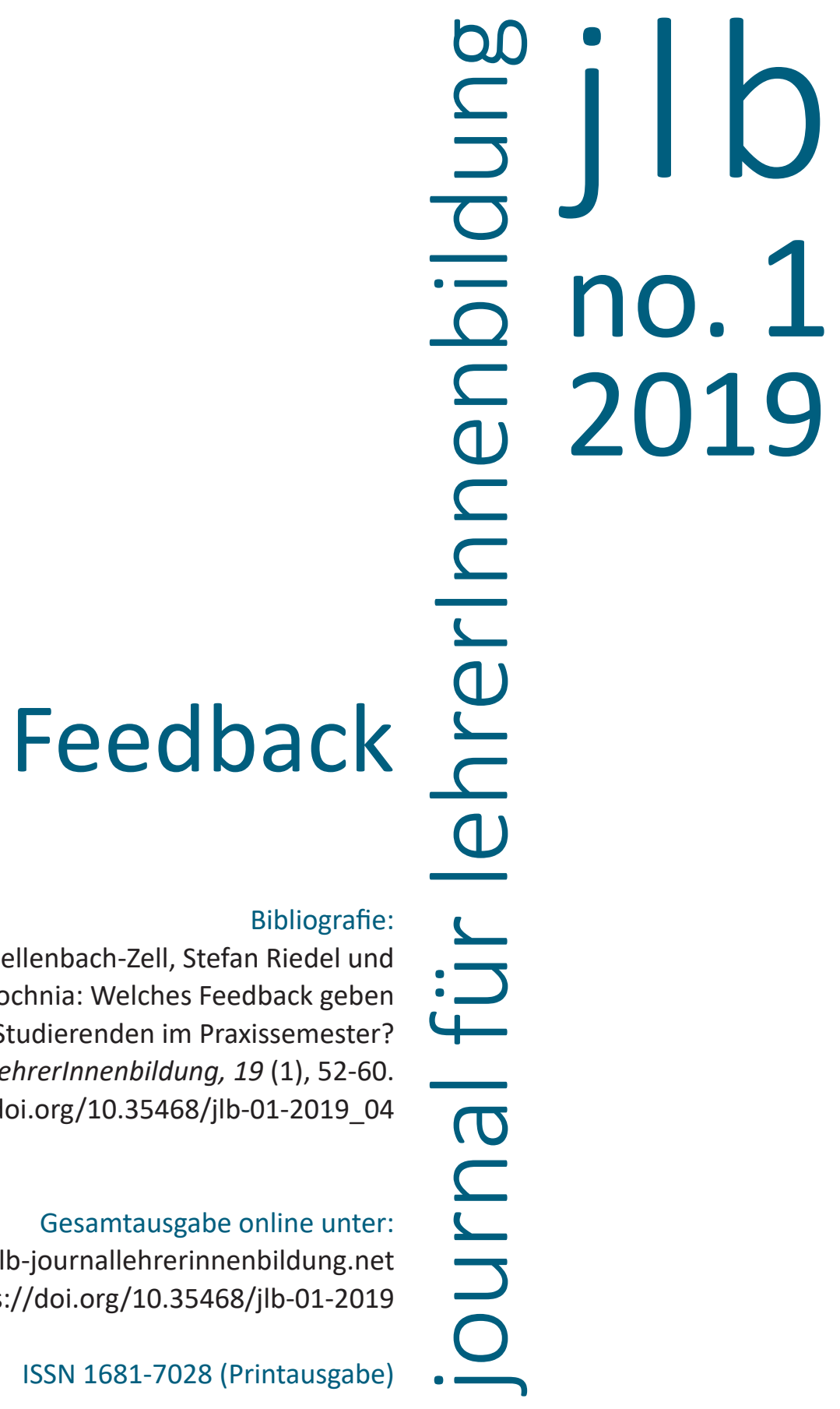


Judith Schellenbach-Zell, Stefan Riedel und Michael Rochnia

\section{Welches Feedback geben Dozent*innen Studierenden im Praxissemester?}




\section{Ziele des Praxissemesters und die Aufgabe der Dozent*innen}

Das Praxissemester stellt für Studierende des Lehramtes in mittlerweile den meisten deutschen Bundesländern eine Lerngelegenheit dar, die den Studierenden ermöglichen soll, theoretische mit praktisch ausgerichteten Studienelementen zu verbinden. Gerade durch die universitäre Einbettung dieser Praxisphase in das fortgeschrittene Studium steht damit eine kriteriengeleitete und forschungsbasierte Durchdringung von praktischen Situationen im Vordergrund (Weyland, 2010). Zentrales Ziel des Praxissemesters ist es, Studierende auf vielfältige Weise zur Reflexion anzuleiten, um Verbindungen zwischen einer konkreten pädagogischen Situation und dem universitär erworbenen Wissen herzustellen. Reflexion meint also eine "bewusste[n] Vermittlung zwischen den Erlebnissen und Erfahrungen einer Lehrkraft in Schule und Unterricht, deren Erwartungen und Überzeugungen sowie dem ihr zur Verfügung stehenden pädagogischen, didaktischen und fachlichen Wissen" (Leonhard \& Rihm, 2011, S. 240). Auf diese Weisen kann eine Anwendung des Wissens unterstützt werden, um der Gefahr entgegenzuwirken, dass das Wissen träge bleibt, wenn universitäre Studieninhalte und schulische Anforderungen unverbunden nebeneinander existieren (Feimann-Nemser, 2001; Renkl, 1996). Damit kommt der universitären Begleitung im Praxissemester die Aufgabe zu, entsprechende Lerngelegenheiten zur Reflexion zu entwickeln und die darauf bezogenen Lernprozesse zu fördern. Bei solchen komplexen Anforderungen wie dem Verknüpfen von Theorie und Praxis spielt das Feedback der Dozent*innen eine maßgebliche Rolle, damit die Studierenden ihren eigenen Fortschritt angemessen einschätzen können. Wir verstehen unter Feedback allgemein die „information communicated to the learner that is intended to modify his or her thinking or behavior for the purpose of improving learning" (Shute, 2008, S. 154). Ein Format, das sowohl Reflexions- als auch Feedbackprozesse berücksichtigt, stellt das sogenannte Lerntagebuch dar, das von den Dozent*innen in der universitären bildungswissenschaftlichen Begleitung eingesetzt wird. Das Lerntagebuch regt die Studierenden dazu an, die Verantwortung für das eigene Lernen und den Lernprozess zu organisieren und stellt darüber hinaus auch die Grundlage der Rückmeldung durch die Dozent*innen dar (Rambow \& Nückles, 2002). Im Rahmen der fünfmonatigen Praxisphase sind die Stu- 
dierenden zu verschiedenen Zeitpunkten angehalten, für sie relevante pädagogische Situationen (z. B. im beobachteten oder selbst durchgeführten Unterricht), denen sie in ihrem Praktikum begegnen, zunächst zu beschreiben und sie anhand ihres wissenschaftlichen Wissens (also Theorien und Befunde) zu erklären und zu analysieren. Aufgabe der Dozent*innen ist es also, Studierenden zu dieser geleisteten TheoriePraxis-Verknüpfung Rückmeldung zu geben. Dabei wird das Feedback der Dozent*innen von den Studierenden durchaus als relevant für die eigene Professionalisierung angesehen (Schellenbach-Zell \& Mertens, angenommen). Fehlen Rückmeldungen zum angestrebten Lernziel, so kann dies zu Unsicherheiten bei den Studierenden bezüglich ihres Lernstandes führen (Ittner \& Hascher, 2016).

Über das Feedback von Dozent*innen bei komplexen Lernprozessen wie der Theorie-Praxis-Verknüpfung während der Praxisphasen ist bisher wenig bekannt. Daher stellt sich ganz explorativ zunächst die Frage im Sinne einer Bestandsaufnahme, zu welchen Kategorien Dozent*innen in einem solchen Rahmen Rückmeldungen geben. Dazu wurde im Rahmen des Projektes „Kohärenz in der Lehrerbildung (KoLBi) ${ }^{1 “}$ ein Teilprojekt durchgeführt, das die Inhalte von Rückmeldungen von Dozent*innen in den Blick nimmt.

\section{Studie}

Zur Untersuchung reichten bei einem Praxissemesterdurchlauf in den letzten zwei Jahren verschiedene Dozent*innen der universitären Begleitung ihr schriftliches Feedback auf 237 Lerntagebücher von Studierenden ein. Dabei handelt es sich um das erste von insgesamt fünf Lerntagebüchern, die die Studierenden im Rahmen des Praxissemesters verfassen. Aus diesen Feedbacks ermittelten wir randomisiert eine Stichprobe von insgesamt 24 Feedbacks. Die Rückmeldungen der Dozierenden wurden im Rahmen einer qualitativen Inhaltsanalyse nach Mayring (2010) induktiv in einem Kategoriensystem klassifiziert und durch eine inhaltlich strukturierende Analyse ausgewertet. Durch diese regelgeleitete Analyse wurden die Rückmeldungen mittels Ge-

1 Das Projekt „Kohärenz in der Lehrerbildung“ (KoLBi) der Bergischen Universität Wuppertal wird im Rahmen der gemeinsamen „Qualitätsoffensive Lehrerbildung“ von Bund und Ländern aus Mitteln des Bundesministeriums für Bildung und Forschung gefördert. Förderkennzeichen: 01JA150. 
neralisierung und Reduktion in ein Kategoriensystem eingeordnet, wodurch eine inhaltliche Strukturierung des Materials erfolgt.

Als Kodiereinheit wird mindestens ein Satzteil mit einer Bedeutungseinheit angesehen. Wenn sich die relevante Information über mehrere Satzteile oder Sätze erstreckt, vergrößert sich die Kodiereinheit entsprechend. Wechselt innerhalb einer Einheit die Information, wird dieser Teil in einer anderen Kategorie untergebracht. Wird eine bereits kodierte Texteinheit (Code) im Text an einer anderen Stelle inhaltlich wiederholt, erfolgt keine Kodierung, um das Ergebnis nicht zu verzerren (Mayring, 2016).

Insgesamt wurden 97 Kodierungen vorgenommen. Im Durchschnitt wurden je Feedback 4,04 Codes zugeordnet. In den gesichteten Feedbacks wurden mindestens zwei und maximal sechs Codes identifiziert. Der Umfang der Rückmeldungen bewegt sich zwischen 51 und 351 Wörtern. Insgesamt ließen sich vierzehn Kategorien ableiten, die in sechs Oberkategorien eingeordnet wurden. Die Ankerbeispiele wurden aufgrund ihres Umfangs gekürzt und paraphrasiert, verdeutlichen jedoch in der Ausrichtung die einzelnen Kategorien:

1. Hinweise auf die Qualität der Aufgabenbearbeitung (Ist-Zustand): Diese Rückmeldungskategorie bezieht sich auf die zu erbringende Leistung in unterschiedlicher Weise. Die Rückmeldungen weisen darauf hin, wie das Feedback verstanden werden soll, sodass Missverständnisse vermieden werden können (1.1: „Mein Feedback bedeutet nicht, dass Sie diesen Lerntagebucheintrag nochmals überarbeiten müssen."). Zudem werden Hinweise auf eine unzureichende Beschreibung der erlebten Unterrichtssituation gegeben (1.2: „Da Sie sich im Anschluss aber auf den Aspekt des Unterrichtsflusses als einen Link zur Reflexion beziehen, braucht der Leser hier noch mehr Informationen."). Die rückgemeldeten Aspekte fordern eine reflexivere Haltung ein (1.3: „Wie bewerten Sie diesen Blickwinkel?") und verdeutlichen, dass die Verknüpfung zwischen Theorie und erlebter Unterrichtssituation forciert werden soll (1.4: „Sie können also durchaus Ihre Beobachtungen noch etwas mehr auf Ihr bildungswissenschaftliches Wissen anwenden."). Zum Teil zielen die Rückmeldungen auf einen stärkeren Theoriebezug ab, in manchen Fällen wird dabei auf konkrete Literatur verwiesen (1.5: „Beim Lesen der Situation erschienen mir folgende weitere Anknüpfungspunkte möglich z. B. hinsichtlich operanter Lerntheorien oder Motivationstheorien."). 
2. Beurteilung des Lerntagebuchs: In dieser Kategorie nehmen Dozent*innen entweder Beschreibungen (2.1: „Sie haben sich auf der Grundlage eines Anlasses mit dem Thema Burnout befasst. Hierzu haben Sie eine Definition entworfen.") oder Beschreibungen in Verbindung mit Wertungen der gezeigten Reflexionsleistung vor (2.2: „Es gefällt mir sehr gut, dass vor allem im Ausblick noch einmal Verbesserungsmöglichkeiten und bereits erfolgreiche Verhaltensweisen gegenüberstellt werden.").

3. Künftige Handlungsoptionen (Ausblick auf den Soll-Zustand): Diese Kategorie fokussiert auf Vorschläge zur Optimierung der Reflexionsleistung in Richtung des kommunizierten Soll-Zustandes. Die rückgemeldeten Aspekte bestärken grundsätzlich den eingeschlagenen Weg (3.1: „Weiter so!"), verdeutlichen didaktische Sichtweisen, die konkret auf die erlebten Unterrichtssituationen Bezug nehmen (3.2: "Ich denke an Teamteaching. vielleicht wäre es ja auch für Sie ein guter Einstieg, nicht gleich eine ganze Stunde planen zu müssen, sondern ,nur' für ausgewählte Fälle differenzierendes Material zu entwickeln."), beinhalten konkrete anforderungsspezifische Empfehlungen bzw. formale Hinweise im Hinblick auf eine zu optimierende Struktur der schriftlichen Reflexion (3.3.1: „Vielleicht können die oben angeführten Leitfragen Ihnen helfen, den Fokus noch stärker auf die Reflexion der Situation zu legen und Ihre Situation kleinteiliger und konkreter zu beschreiben.") oder liefern Hinweise, welche die Form des eingereichten Lerntagebuchs betreffen und auf eine strukturiertere Darstellungsleistung abzielen (3.3.2: „Anbei erhalten Sie einen neuen Bogen für den Eintrag Ihrer Lerntagebücher. Bitte benutzen Sie diesen für die nächsten Einträge. Dieser gibt eine Struktur vor, die Ihnen bei der Erstellung Ihrer Einträge und der Reflexion helfen soll.").

4. Die Kategorie Lob beinhaltet aufmunternde Worte mit einem allgemeinen Bezug zur Aufgabe (,/hr Lerntagebucheintrag Nr. 1 ist insgesamt gelungen. Er zeugt von Reflexivität.")

5. Ableitung für den Professionalisierungsprozess: Diese Kategorie umfasst Hinweise und Kommentare zur Weiterentwicklung des eigenen Professionalisierungsprozesses. (,Sie haben deutlich gezeigt, inwieweit das Thema für Sie selbst und Ihre professionelle Entwicklung relevant ist, und Sie konnten aus der Reflexion für Sie bedeutsame weitere Themen ableiten, mit denen Sie sich beschäftigen möchten.") 
6. Positive Erwartung: Diese Kategorie rückt die Beziehungsebene zwischen den Dozent*innen und den Studierenden in den Vordergrund und drückt positive Erwartungen bezüglich künftiger Leistungen aus. (,Ich freue mich auf Ihr nächstes LTB!")

Die zugeordneten Codes werden noch einmal quantifiziert in Tabelle 1 dargestellt, um Häufigkeiten und entsprechende Gewichtungen zu verdeutlichen. Insgesamt lässt sich konstatieren, dass sich die Dozent*innen in der Hauptsache in ihren Feedbacks auf die verschiedenen Facetten der Bearbeitungsqualität und auf Möglichkeiten der Handlungsoptionen Bezug nehmen, bzw. die aktuelle Leistung beschreiben und bewerten.

Tab. 1 Häufigkeiten der zugeordneten Codes $(\Sigma=97)$

\begin{tabular}{|c|c|}
\hline Identifizierte Hauptkategorien & $\begin{array}{c}\text { Verteilung } \\
\text { der Zuord- } \\
\text { nungen }\end{array}$ \\
\hline \multicolumn{2}{|l|}{ (1) Hinweise auf die Qualität der Aufgabenbearbeitung (Ist-Zustand) } \\
\hline (1.1) Hinweise zum Umgang mit dem Feedback & $2,06 \%$ \\
\hline (1.2) Unzureichende Situationsbeschreibung & $11,34 \%$ \\
\hline (1.3) Reflexive Haltung & $2,06 \%$ \\
\hline (1.4) Verknüpfung von Theorie und Situation & $3,09 \%$ \\
\hline (1.5) Stärkung Theoriebezug & $12,37 \%$ \\
\hline
\end{tabular}

(2) Beurteilung des Lerntagebuchs

(2.1) Beschreibung

$6,19 \%$

(2.2) Beschreibung und Bewertung

$18,56 \%$

(3) Künftige Handlungsoptionen (Ausblick auf den Soll-Zustand)

\begin{tabular}{|c|c|c|}
\hline & (3.1) Allgemeine Bekräftigung & $4,12 \%$ \\
\hline & (3.2) Situationsbezogene Handlungsempfehlungen & $7,22 \%$ \\
\hline & (3.3.1) Anforderungsspezifische Handlungsempfehlungen & $8,25 \%$ \\
\hline & (3.3.2) Handlungsempfehlungen zu Struktur und Formalität & $5,15 \%$ \\
\hline (4) & Lob & $11,34 \%$ \\
\hline (5) & Ableitung für den Professionalisierungsprozess & $6,19 \%$ \\
\hline (6) & Positive Erwartung & $2,06 \%$ \\
\hline
\end{tabular}




\section{Einordnung der Ergebnisse}

Die Ergebnisse zeigen, dass es den Dozent*innen zu weiten Teilen gelungen ist, effektives Feedback zu geben. Sie berücksichtigen die Ebenen (task level, process level, selfregulation level, self level), die sich in der Beantwortung der drei Fragen zeigen, die die Forschung für effektives Feedback als maßgeblich ansieht: (1) where am I going? (2) how am I going? und (3) where to next? (Hattie \& Gan, 2011). Die Dozent*innen geben Hinweise darauf, wie die Aufgabe der Studierenden, praktische Situationen mit Theorien oder Befunden zu verknüpfen, umgesetzt wurde und bewegen sich damit auf dem task level. Darüber hinaus beziehen die Dozent*innen in ihr Feedback auf dem process und dem selfregulation level auch Empfehlungen ein, was Studierende tun können, um die Leistung zu verbessern - auch auf formaler Ebene. Das self level, also Rückmeldungen rein zur Person und ihren Fähigkeiten, wird durch die Dozent*innen kaum bedient. Gerade Kategorie (3) macht die Heterogenität der Rückmeldungen deutlich, indem sie sowohl ganz situationsspezifische aber auch anforderungsspezifische Handlungsempfehlungen umfasst. Dieser Punkt verweist u. E. auf die Rollenunklarheit bei den Dozent*innen verbunden mit der Frage, welcher Auftrag Dozent*innen in einem komplexen Zusammenspiel mit weiteren Institutionen des Schulkontextes zukommt. Insgesamt lässt sich jedoch festhalten, dass die Beurteilung einer Aufgabe wie der Verknüpfung von Theorie und Praxis und entsprechendes Feedback auch für Dozent*innen eine voraussetzungsreiche Anforderung darstellt. Dabei ist es erforderlich, dass sie über Wissen und Operationalisierungen auf verschiedenen Ebenen verfügen. So müssen sie auf inhaltlicher Ebene zum einen wissen, welches wissenschaftlich abgesicherte Wissen zu einer bestimmten Situation überhaupt zur Verfügung steht, aber auch, wie sie die Verknüpfungsleistung der Studierenden einschätzen können. Nicht zuletzt müssen Dozent*innen außerdem Wissen darüber erworben haben, wie sie effektives Feedback gestalten können, um weitere Lernprozesse zu ermöglichen. In diesem breiten Aufgabenfeld ist es von Hochschulseite denkbar, Dozent*innen (insbesondere diejenigen mit wenig Erfahrungen) zusätzlich mit Workshops zu Meta-Themen wie der Einschätzung der Theorie-Praxis-Verknüpfungen und zu effektivem Feedback zu unterstützen. Feedback-Leitfäden, die sich sowohl auf die Theorie- 
Praxis-Verknüpfung und auch auf die Beantwortung der drei oben genannten Fragen beziehen, können passend dazu einen zusätzlichen Beitrag leisten und werden derzeit im KoLBi-Projekt auf ihre Wirkung hin überprüft.

\section{Literatur}

Feimann-Nemser, S. (2001). From Preparation to Practice: Designing a Continuum to Strengthen und Sustain Teaching. Teachers College Record, 103, 1013-1055.

Hattie, J. \& Gan, M. (2011). Instruction Based on Feedback. In P. A. Alexander \& R. E. Mayer (Eds.), Handbook of Research on Learning and Instruction (pp. 249271). New York: Routledge.

Ittner, D. \& Hascher, T. (2016). Zur Rolle des Feedbacks für das Lehren und Lernen mit Portfolios im Hochschulkontext. In S. Ziegelbauer \& M. Gläser-Zikuda (Hrsg.), Das Portfolio als Innovation in Schule, Hochschule und LehrerInnenbildung (S. 13-25). Bad Heilbrunn: Verlag Julius Klinkhardt.

Leonhard, T. \& Rihm, T. (2011). Erhöhung der Reflexionskompetenz durch Begleitveranstaltungen zum Schulpraktikum? - Konzeption und Ergebnisse eines Pilotprojektes mit Lehramtsstudierenden. Lehrerbildung auf dem Prüfstand, 4 (2), 240270.

Mayring, P. (2010). Qualitative Inhaltsanalyse: Grundlagen und Techniken (11. akt. u. überarb. Aufl.). Weinheim: Beltz.

Mayring, P. (2016). Einführung in die qualitative Sozialforschung (6. überarb. Aufl.). Weinheim: Beltz Verlag.

Rambow, R. \& Nückles, M. (2002). Einsatz des Lerntagebuchs in der Hochschullehre. Das Hochschulwesen, 50 (3), 113-120.

Renkl, A. (1996). Träges Wissen: Wenn Erlerntes nicht genutzt wird. Psychologische Rundschau, 47 (2), 78-92.

Schellenbach-Zell, J. \& Mertens, S. (angenommen). Kohärente Lernziele und ihre Effekte auf die Bewertung von Lerngelegenheiten im Praxissemester. In BMBF (Hrsg.), Verzahnung von Theorie und Praxis im Lehramtsstudium. Erkentnisse aus den Projekten der "Qualitätsoffensive Lehrerbildung“.

Shute, V. (2008). Focus on Formative Feedback. Review of Educational Research, 78 (1), 153-189.

Weyland, U. (2010). Zur Intentionalität schulpraktischer Studien im Kontext universitärer Lehrerausbildung. Paderborn: Eusl. 
Judith Schellenbach-Zell, Dr., wiss. Mitarbeiterin am

Institut für Bildungsforschung an der Bergischen Universität Wuppertal.

Arbeitsschwerpunkte: Praxisphasen in der Lehrer*innenbildung, Begleitformate in der Lehrer*innenbildung zur Theorie-Praxis-Verzahnung

zell@uni-wuppertal.de

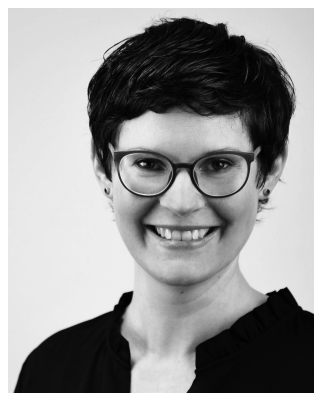

Stefan Riedel, Studienreferendar am Zentrum für schulpraktische Lehrer*innenausbildung in Hagen.

Arbeitsschwerpunkt: Feedback und Mentoring

\section{steriebkh@googlemail.com}

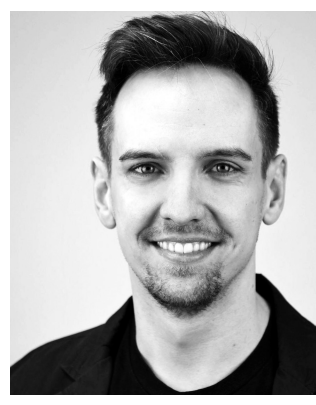

Michael Rochnia, wiss. Mitarbeiter am Institut für Bildungsforschung an der Bergischen Universität Wuppertal.

Arbeitsschwerpunkte: Nutzung von Evidenz in pädagogischen Bereichen, Interkulturalität im Politikunterricht

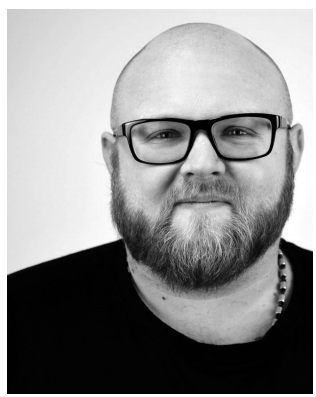

